\title{
Extreme gas properties in the most distant quasars
}

\author{
R. Maiolino ${ }^{1}$, E. Oliva ${ }^{1,2}$, F. Ghinassi ${ }^{2}$, M. Pedani ${ }^{2}$, F. Mannucci ${ }^{3}$, R. Mujica ${ }^{4}$, and Y. Juarez ${ }^{4}$ \\ 1 INAF - Osservatorio Astrofisico di Arcetri, Largo E. Fermi 5, 50125 Firenze, Italy \\ 2 INAF - Telescopio Nazionale Galileo, Calle Alvarez de Abreu, 70, 38700 Santa Cruz de La Palma, Spain \\ 3 Istituto di Radioastronomia, sezione di Firenze - Largo E. Fermi 5, 50125 Firenze, Italy \\ ${ }^{4}$ Instituto Nacional de Astrofísica Óptica y Electronica, Puebla, Luis Enrique Erro 1, Tonantzintla, Puebla 72840, Mexico
}

Received 18 November 2003 / Accepted 15 March 2004

\begin{abstract}
We present near-IR, low resolution spectra of eight of the most distant quasars known, with redshifts in the range $4.9<z<6.4$. Half of these quasars are characterized by deep, broad and blueshifted absorption features associated with both high and low ionization species (CIV, SiIV, AlIII, MgII), i.e. they belong to the class of Broad Absorption Line (BAL) quasars, which are associated with powerful outflows of dense gas. Although the sample is small, the large fraction of BAL quasars, the depth and ionization state of the absorption features suggest that these most distant quasars are surrounded by a much larger amount of dense gas than lower redshift $(z<4)$ quasars. The possible interpretation in terms of extremely high accretion rates and the association with the early formation of quasars and of their host galaxies is discussed. The absorption properties of the dust, associated with the gas along the line of sight, appear different with respect to lower redshift quasars, possibly indicating different dust physics at these highest redshifts.
\end{abstract}

Key words. galaxies: active - galaxies: evolution - galaxies: high-redshift - galaxies: quasars: absorption lines galaxies: quasars: general

\section{Introduction}

The exceptional luminosities of quasars allow us to study the properties of gas and dust in their circumnuclear region even in the most distant systems known. The strong emission lines excited by the nuclear source have been widely used to infer the metallicity and the physical/dynamical state of the gas (e.g., Hamman \& Ferland 1999; Verner et al. 2003). These studies have been extended to the most distant quasars known at $z>5$, approaching the epoch of re-ionization and of the formation of the first generation of stars. The UV rest-frame emission line properties of the highest redshift quasars have indicated little or no evolution of the gas metallicity (e.g., Dietrich et al. 2003; Maiolino et al. 2003; Pentericci et al. 2002) up to $z=6.4$, suggesting that the first epoch of star formation occurred at $z>$ 9 , in these objects. Recent sub-mm and mm observations have allowed the detection of dust emission in some quasars at $z>5$, indicating that large amounts of dust were already formed at these early epochs (Bertoldi et al. 2003; Priddey et al. 2003).

Within this context, Broad Absorption Line (BAL) quasars offer additional tools to investigate the circumnuclear medium. These objects, which account for about $15 \%$ of the whole quasar population at low and intermediate redshifts (Reichard et al. 2003b; Hewett \& Foltz 2003), are characterized by deep and broad absorption features associated with UV

Send offprint requests to: $\mathrm{R}$. Maiolino,

e-mail: maiolino@arcetri.astro.it resonant lines. Generally the most prominent absorption line is CIV $\lambda 1549$. A small fraction of BAL quasars (16\%, or $\sim 2 \%$ of all quasars at low and intermediate redshifts) also show absorption by low ionization species (MgII $\lambda 2798$ and often AlIII] 21857 ), these are called LoBALs (in contrast to HiBALs, which only show the high ionization absorption lines). An even rarer class are FeLoBALs, which also show prominent FeII and FeIII absorption. The basic interpretation is that in BAL quasars we are seeing the active nucleus through a dense outflowing gas. BALs are characterized by redder continua with respect to non-BALs, which is commonly interpreted as reddening by dust associated with the outflowing gas (Reichard et al. 2003b; Brotherton et al. 2001). The reddening is generally fit best by a SMC-like extinction curve, at variance with more absorbed AGNs which are characterized by a flatter extinction curve (Gaskell et al. 2003; Maiolino et al. 2001a,b). LoBALs are generally characterized by higher reddening than HiBALs (Reichard et al. 2003b; Becker et al. 2000).

Various interpretations have been given for the BAL phenomenon. One possibility is that all quasars are characterized by an outflowing wind, but occurring only in certain preferential directions; within this scenario BALs and non-BALs are the same kind of quasars but observed along different viewing angles (Weymann et al. 1991; Elvis 2000; Ogle et al. 1999; Schmidt \& Hines 1999). Alternatively, BALs and nonBALs could be two distinct populations of quasars (Surdej \& Hutsemekers 1987; Boroson \& Meyers 1992), with and without 
outflows. Becker et al. (2000) suggested that BALs may trace the early stages of quasar activity, while King (2003) and King \& Pounds (2003) suggested that strong winds may be related to episodes of enhanced accretion.

We have observed in the near-IR (UV rest-frame) a sample of eight quasars in the redshift range $4.9<z<6.4$ (among the highest redshift quasars currently known). The spectra were obtained with an instrumental setup that can cover the full near-IR spectrum from $0.8 \mu \mathrm{m}$ to $2.4 \mu \mathrm{m}$ in a single exposure (and with very high throughput). We find that four of these objects are BALs, and two (probably three) of them are LoBALs. Although the statistics are very limited, this result suggests that the fraction of BALs and LoBALs at $z>5$ may be higher than at lower redshift. We discuss the extreme properties of the gas and dust absorption in these objects, in comparison with lower redshift quasar. We infer that gas and dust in these most distant quasars are likely to be different with respect to the lower redshift cases, and this is probably related to the early evolutionary stage of these quasars and of their host galaxies.

\section{Observations and data reduction}

The observations were obtained with the Near Infrared Camera Spectrograph (NICS) at the Italian Telescopio Nazionale Galileo (TNG), a $3.56 \mathrm{~m}$ telescope. Among the various imaging and spectroscopic observing modes (Baffa et al. 2001), NICS offers a unique, high sensitivity, low resolution observing mode, which uses an Amici prism as a dispersing element (Oliva et al. 2003). In this mode it is possible to obtain the spectrum from $0.8 \mu \mathrm{m}$ to $2.4 \mu \mathrm{m}$ in a single exposure. The throughput of the Amici prism is nearly two times higher than other more commonly used dispersers. The spectral resolution with a $0.75 \mathrm{slit}$, as it was in our case, is $75\left(4000 \mathrm{~km} \mathrm{~s}^{-1}\right)$ and nearly constant over the whole wavelength range. This observing mode is appropriate to study the near-infrared continuum of faint sources and to detect broad $\left(\geq 4000 \mathrm{~km} \mathrm{~s}^{-1}\right)$ emission and absorption lines in faint quasars.

Observations were performed in three observing runs: 2002 November 7-9; 2003 February 25-29; and 2003 May 23-26. Several quasars were observed more than once on different nights to check for any instrumental or observational artifacts in the individual spectra. Wavelength calibration was performed by using an argon lamp and the deep telluric absorption features. The telluric absorption was then removed by dividing the quasar spectrum by a reference star spectrum (FV-GV) observed at similar airmass. The intrinsic features and slope of the reference star were then removed by multiplying the corrected quasar spectrum by a spectrum of the same stellar type from the library by Pickles et al. (1998), smoothed to our resolution. Absolute flux calibration was obtained by using the photometry on the acquisition image or through photometry reported in the literature.

These spectra were also discussed in Maiolino et al. (2003) in relation to the FeII bump and MgII emission, with the goal of measuring the iron abundance as a function of redshift. Here we focus on the detection of the UV absorption lines tracing BALs and on the continuum shape. For this reason we restrict this investigation to the quasars in the Maiolino et al. (2003) sample with $z>4.9$, for which any putative CIV absorption is redshifted into the spectral range where Amici+NICS allows a reliable detection (for all the quasar at lower redshift the spectrum on the blue side of CIV is too noisy to infer the presence of any absorption). We include also the data of SDSSJ1044-0125 obtained previously at the same instrument and already published in Maiolino et al. (2001c). The eight quasars selected with this redshift constraint are all from the Sloan Digital Sky Survey (SDSS).

The quasars in our sample are listed in Table 1, where their full IAU designations are reported. However, in the following we will adopt a shortened version of their names.

\section{BAL quasars}

In this sample we identify four high redshift BAL quasars, which are discussed in detail in this section.

We determine the continuum slope and the balnicity index of the CIV absorption (which is similar to the Equivalent Width expressed in $\mathrm{km} \mathrm{s}^{-1}$, as defined in Weymann et al. 1991) by following the method described in Reichard et al. (2003a). More specifically, the average SDSS non-BALs quasar spectrum, which has a continuum slope $\alpha=-1.61\left(F_{\lambda} \propto \lambda^{\alpha}\right)$, is taken as a template and modified to match the observed spectrum. The template is smoothed to our resolution. Then the continuum shape is modified to match the line-free regions of the observed spectrum either by changing the spectral index (by multiplying the spectrum by $\lambda^{\alpha /-1.61}$ ) or by absorbing the template with an SMC extinction curve. The CIV emission intensity of the template may also be modified to match the observed intensity and to better calculate the balnicity index; in practice in our cases this is a very difficult task, since the CIV emission is so heavily absorbed that it is difficult to estimate its intrinsic flux and profile (this issue is discussed more in detail below).

\subsection{SDSS1048+46: The most distant LoBAL quasar $(z=6.22)$}

At a redshift of 6.22 this quasar is now the highest redshift BAL known. Fan et al. (2003) suggested that, based on a hint of SiIV absorption in the optical spectrum, this might be a BAL. The Amici+NICS spectrum is shown in Fig. 1a and clearly displays a very deep and very broad CIV absorption. The absorption profile is resolved and displays a clear double trough, a feature which is commonly observed in LoBALs. The two main troughs have a blueshift of 11000 and $20000 \mathrm{~km} \mathrm{~s}^{-1}$, which are labeled as systems 1 and 2, respectively, in Fig. 1a. At the lower velocity shift $\left(11000 \mathrm{~km} \mathrm{~s}^{-1}\right)$ there is also a deep SiIV absorption and absorption of AlIII] and MgII, which identify this object as a LoBAL.

In Fig. 1a the thin solid line shows the non-BAL template where the slope has been adapted to match the line-free continuum regions (redward of CIV). We have to force a slope of $\alpha=-2.1$, bluer than the average SDSS spectrum (but in agreement with the slope of dereddened BALs, as discussed below). There is a strong mismatch between the template and the observed spectrum blueward of CIV which might be ascribed to the very deep absorption by the UV lines (but see below for an 
Table 1. Summary of the properties derived from the spectra of our sample.

\begin{tabular}{lllllll}
\hline \hline Name & $z$ & Type & $B I(\mathrm{CIV})^{a}$ & $\alpha^{b}$ & $E(B-V)^{c}$ & $M_{i^{*}}{ }^{b}$ \\
\hline SDSSJ075618.14+410408.6 & $5.08^{e}$ & BAL $^{f}$ & $270 \pm 60^{g}$ & -1.67 & - & -26.6 \\
SDSSJ103027.10+052455.0 & 6.28 & & & -1.55 & 0.001 & -28.1 \\
SDSSJ104433.04-012502.2 & $5.78^{h}$ & HiBAL & $1950 \pm 250^{g}$ & -1.55 & 0.001 & -28.4 \\
SDSSJ104845.05+463718.3 & $6.22^{i}$ & LoBAL & $6500 \pm 1100^{g}$ & -2.10 & $-j$ & -27.4 \\
SDSSJ114816.64+525150.3 & 6.40 & & & -1.70 & - & -28.5 \\
SDSSJ130608.26+035626.3 & 5.99 & & & -1.80 & - & -27.8 \\
SDSSJ160501.21-011220.6 & 4.92 & LoBAL & $9300 \pm 2000^{g}$ & -1.35 & 0.03 & -27.7 \\
SDSSJ173744.88+582829.6 & $4.88^{k}$ & & & -2.10 & - & -26.9 \\
\hline
\end{tabular}

Notes:

${ }^{a}$ Balnicity index of the CIV absorption as defined in Weymann et al. (1991), which is "similar" to the Equivalent Width of the absorption line measured in $\mathrm{km} \mathrm{s}^{-1}$.

${ }^{b}$ Slope of the continuum $\left(F_{\lambda} \propto \lambda^{\alpha}\right)$; this is calculated by comparison with the non-BAL SDSS template which has $\alpha=-1.61$ (see text).

${ }^{c}$ Reddening to apply to the SDSS non-BAL template to match the observed spectrum; a SMC extinction curve is assumed (see text).

${ }^{d}$ Absolute magnitude in the rest frame $i^{*}$ band, obtained by using the observed spectral slope and assuming $H_{0}=50 \mathrm{~km} \mathrm{~s}^{-1} \mathrm{Mpc}^{-1}, \Omega_{\mathrm{m}}=1$ and $\Omega_{\Lambda}=0$ (to allow for a direct comparison with Reichard et al. 2003a); it is not corrected for intrinsic absorption of the QSOs.

${ }^{e}$ This is the redshift obtained by our spectrum, while the redshift reported in the literature is 5.09.

${ }^{f}$ Possible FeLoBAL (see text).

${ }^{g}$ All errors on the balnicity indices are dominated by the uncertainty on the profile of the CIV emission.

${ }^{h}$ This is the redshift obtained by our spectrum, while Goodrich et al. (2001) report a redshift of 5.74.

${ }^{i}$ This is our best estimate of the redshift based on the MgII (which is slightly different than the redshiftof 6.23 published by Fan et al. 2003); however, the CIV and SiIV emission features may suggest an even lower redshift.

${ }^{j}$ The extreme UV continuum of this LoBAL may be fitted with dust reddening, but with an extinction curve totally different with respect to the SMC one adopted for all other BAL (see text for details).

${ }^{k}$ This is the redshift obtained by our spectrum, while the redshift reported in the literature is significantly different: $z=4.94$.

alternative explanation). The continuum slope redward of CIV would indicate that this BAL is not subject to any dust reddening. This result is at odds with what observed in lower redshift BALs, and specifically LoBALs, which are systematically reddened. To illustrate this difference the thin dashed line in Fig. 1a shows the average LoBAL spectrum obtained by the SDSS (Reichard et al. 2003a), which is clearly much redder than the spectrum of SDSS1048+46. All LoBALs quasars observed at lower redshift have an observed spectral slope redder than observed in SDSS1148, more specifically at $z<4$ all LoBAL quasars in the SDSS have an observed $\alpha>-1.9$.

Another possible explanation to fit the observed spectral shape would be to assume that the flattening blueward of $1600 \AA$ is not due to broad line absorption but to dust reddening. However, a SMC extinction curve does not work, since it would make the whole spectrum redder. Even steeper extinction curves, as those inferred in some rare BALs, would be unable to reproduce the observed continuum shape. The only possibility would be a (speculative) extinction curve which is relatively flat at $\lambda>1500 \AA$ and steeply rising at $\lambda<1500 \AA$ (never observed so far). We do not discuss in detail this possibility, since it will be investigated more in detail in a forthcoming paper (Maiolino et al., in prep), but in Fig. 1b we only show the effect of such an unusual extinction curve on the continuum shape. Obviously tackling this issue will require higher spectral resolution over the whole band $0.8-1.3 \mu \mathrm{m}$ to disentangle the effects of broad absorption lines from the true continuum curvature.
On the the very blue spectral shape redward of $1500 \AA$, with $\alpha=-2.1$ it is most interesting to note that the "intrinsic" LoBALs spectral index inferred by Reichard et al. (2003b) is -2.01 (altough there is some degeneracy with reddening). Based also on the analysis of their emission line properties, Reichard et al. (2003b) suggested that BALs may, in fact, be (intrinsically) bluer than the average quasar, despite being dust reddened. The lack of dust absorption (at least reward of $1500 \AA$ ) in SDSS1048+46 allows us to observe directly the intrinsic slope of this BAL and the resulting very blue slope strongly supports the finding of Reichard et al. (2003b) indirectly inferred for lower redshift BALs QSOs.

The balnicity index of CIV cannot be measured very accurately, because it is not possible to properly recover the intrinsic profile of the CIV emission prior of absorption. For this reason we derive a balnicity index with a relatively large error, which is not due to signal-to-noise but dominated by the uncertainty on the CIV emission profile. We obtain: $B I(\mathrm{CIV})=$ $6500 \pm 1100 \mathrm{~km} \mathrm{~s}^{-1}$. As discussed in Sect. 6, this high value is located on the high balnicity indices tail of the BALs observed at lower redshift (Reichard et al. 2003a).

Another interesting feature of this BAL is the presence of absorption even on the red side of CIV (system \#3 in Fig. 1). If associated with redshifted CIV the corresponding receding velocity would be about $-3800 \mathrm{~km} \mathrm{~s}^{-1}$, such a redshifted trough was never observed at lower redshift (only a weak "droop" was found on the red wing of CIV of the average spectrum of BAL quasars by Reichard et al. (2003b), but only after 

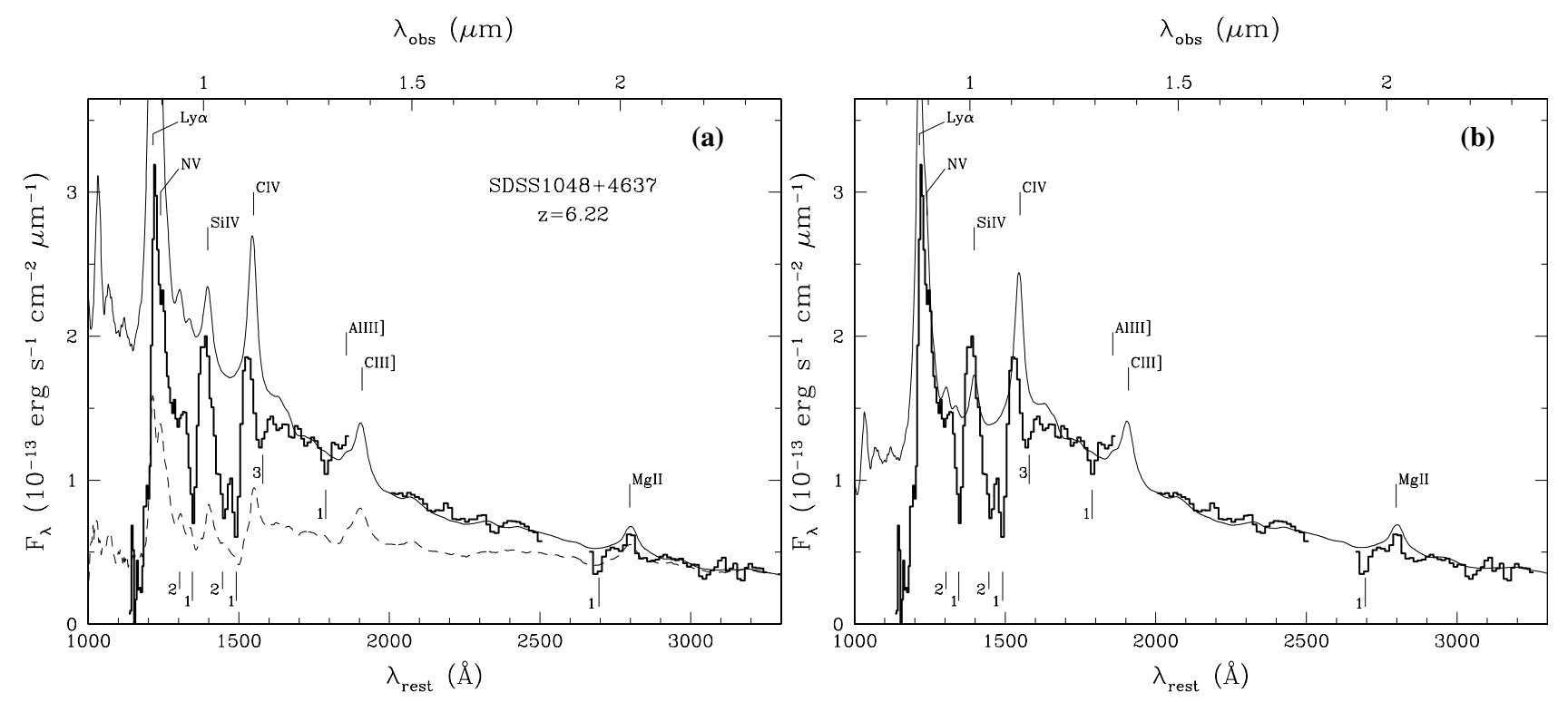

Fig. 1. a) Amici+Nics spectrum of SDSS1048+46, a LoBAL at $z=6.22$ (thick solid line). The thin line shows the non-BAL SDSS template adapted to a slope $\alpha=-2.1$. The thin dashed line shows the average spectrum of LoBAL quasars in the SDSS. The main emission lines are marked. The lower ticks mark the blueshifted absorptions at $11000 \mathrm{~km} \mathrm{~s}^{-1}$ and at $20000 \mathrm{~km} \mathrm{~s}^{-1}$ (systems \#1 and \#2 respectively). System \#3 could be a redshifted absorption feature of CIV at $-3800 \mathrm{~km} \mathrm{~s}^{-1}$, alternatively it could be absorption by HeII $\lambda 1640$ associated with system \#1 (see text). b) Same as a), but where the template is absorbed by an extinction curve which is much steeper in the extreme UV (see text).

a careful subtraction of the emission line profile). The redshifted trough may be explained in a scenario of a rotating disk wind whose size (or at least part of it) is comparable with the continuum emitting region. A similar scenario was invoked by Hall et al. (2002) to explain the suppression of the red wing of CIV in lower redshift BALs. However, we note that the redshifted absorption may well be tracing inflowing gas along the line of sight, again under the assumption that the size of the absorber is comparable with the emitting source. An alternative explanation is that system \#3 is actually absorption of HeII $\lambda 1640$ associated with system \#1. Absorption by HeII would require extreme gas properties in terms of opacity. However, recently HeII blueshifted absorption has been detected in one SDSS quasar (Hall et al. 2004). Higher spectral resolution observations of this object are certainly required to further investigate the nature of this absorption system.

Finally we note that the SiIV and CIV emission lines appear significantly blueshifted with respect to the template at $z=6.22$, which may suggest a lower redshift for this source. However higher spectral resolution is required to better disentangle absorption and emission components and to better determine the redshift of this system. If CIV, in particular, is really blueshifted with respect to the rest frame, this may be consistent with the intrinsically bluer continuum of this QSO, in keeping with the finding by Reichard et al. (2003b) that bluer QSOs tend to be associated with blueshifted CIV.

\subsection{SDSS1605-01: An extreme LoBAL at $z=4.92$}

The Amici+NICS spectrum of this extreme BAL is shown in Fig. 2. This has the deepest CIV absorption of all BALs found at high redshift. Its balnicity index of about $9300 \pm 2000 \mathrm{~km} \mathrm{~s}^{-1}$

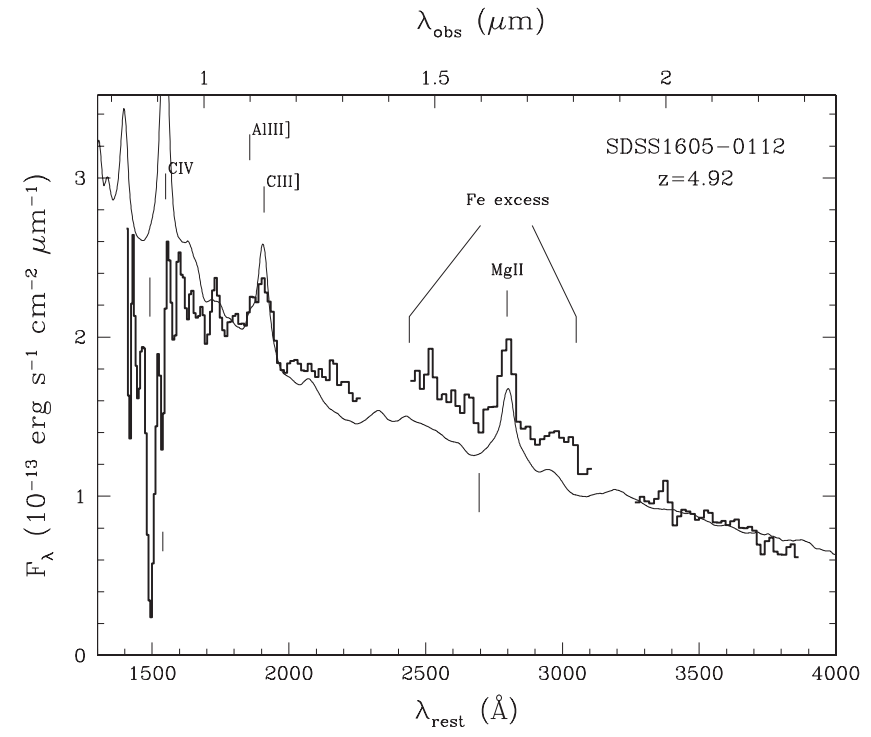

Fig. 2. Amici+Nics spectrum of SDSS1605-01, a LoBAL at $z=4.92$ (thick solid line). The thin line shows the non-BAL SDSS template reddened with an SMC extinction curve with $E_{B-V}=0.03$. The main emission lines are marked (though CIV is essentially totally "eaten" by the absorption). The long ticks mark the blueshifted absorptions at $11000 \mathrm{~km} \mathrm{~s}^{-1}$ for CIV (the deepest trough) and MgII. The shorter thick marks a shallower absorption trough blueshifted by $2000 \mathrm{~km} \mathrm{~s}^{-1}$. Note an excess of FeII emission, not uncommon among the most extreme BALs.

is among the highest value ever found among BALs known so far (again, the large error on the balnicity index is dominated by the large uncertainty on the CIV emission profile). The CIV absorption is so deep that it has "eaten" nearly all of the CIV emission. Balnicity indices higher than $8000 \mathrm{~km} \mathrm{~s}^{-1}$ are 


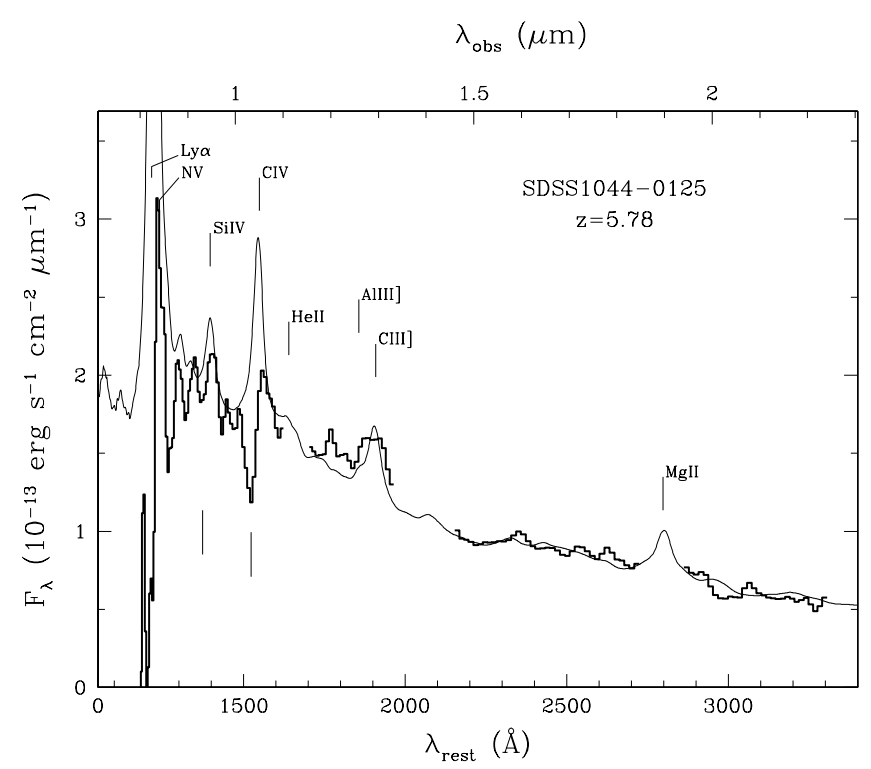

Fig. 3. Amici+Nics spectrum of SDSS1044+01, a HiBAL at $z=5.78$ (thick solid line). The thin line shows the non-BAL SDSS template adapted. The main emission lines are marked. The lower ticks mark the blueshifted absorptions at $5000 \mathrm{~km} \mathrm{~s}^{-1}$.

only found among LoBAL quasars. Indeed, also in this object there is indication of MgII absorption (though with a low $\mathrm{S} / \mathrm{N}$ ), confirming the LoBAL nature of this object.

This is the only BAL clearly characterized by a redder slope than the average of quasars. The thin line in Fig. 2 shows the non-BAL template reddened with an SMC extinction curve to match the observed continuum slope. A reddening of $E_{B-V}=0.03$ is required. Alternatively the spectrum can be fitted by forcing the template to have a flatter power law and, more specifically $\alpha=-1.35$.

The fit in Fig. 2 suggests a strong excess of FeII emission. This excess is unlikely to be a flux calibration problem, both because the observing mode adopted by us prevents this kind of calibration problems between the IR bands (since the full spectrum is obtained simultaneously), and because this object was observed in two different nights and both spectra show the same feature. As noted by Weymann et al. (1991), Fe emission in excess of the average is commonly associated with BAL quasar with high balnicity index.

Finally, we note that this object may also be characterized by redshifted absorption, similar to the previous case. However, higher resolution observations are required to clearly disentangle emission features from possible redshifted absorption troughs.

\subsection{SDSS1044-01: The most distant HiBAL quasar $(z=5.78)$}

The Amici+NICS spectrum of this quasar was already shown in Maiolino et al. (2001c). We present again the spectrum here to extract the continuum slope and balnicity index with the same template spectrum as the other quasars. In Fig. 3 we show the observed spectrum along with the SDSS non-BAL template (thin line). Our spectrum is best fit by a redshift of 5.78 rather

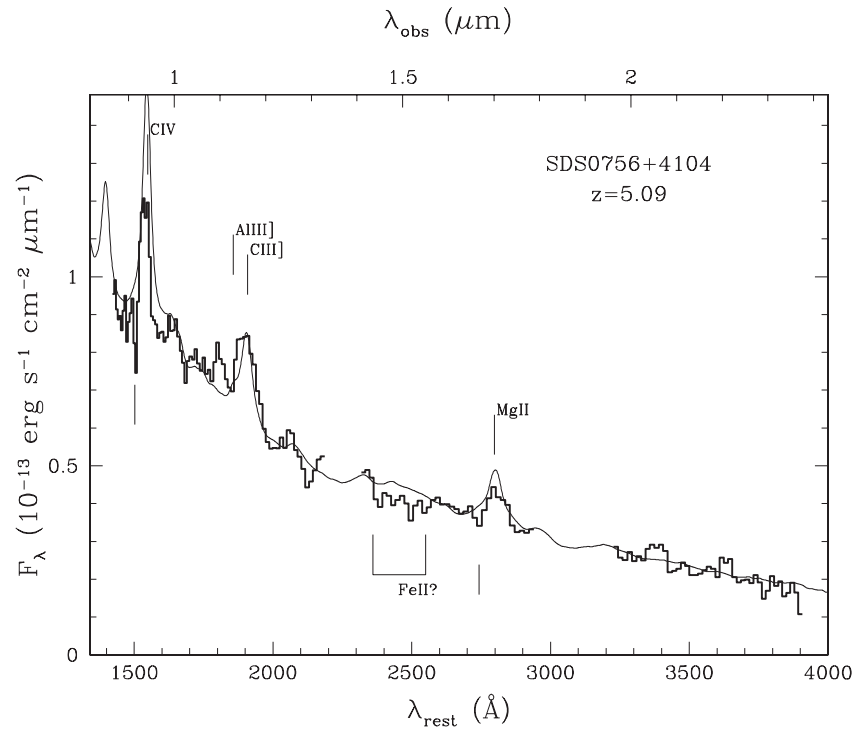

Fig. 4. Amici+Nics spectrum of SDSS0756+41, a BAL at $z=5.09$ (thick solid line). The thin line shows the non-BAL SDSS template adapted. The main emission lines are marked (though CIV and CIII] show an apparent blueshift with respect to the template). The lower ticks mark a CIV blueshifted absorption at $9000 \mathrm{~km} \mathrm{~s}^{-1}$ and a possible MgII absorption blueshifed by $6000 \mathrm{~km} \mathrm{~s}^{-1}$. Possible absorption by FeII is also marked.

than 5.74 derived previously, but the difference my be caused by the blending with the absorption features. The continuum slope appears only slightly redder than the average of the SDSS non-BAL quasars: $\alpha=-1.55$ (though consistent with the unreddened value of -1.62 within the uncertainties). Note that in Maiolino et al. (2001c) we found a better match (both for the continuum and for the emission lines) with the QSO template obtained by Francis et al. (1991). However, even the slope of -1.55 is still significantly bluer than the average slope found in HiBAL quasars at lower redshift, $\langle\alpha(\operatorname{HiBAL}, z<4)\rangle=$ -1.39 , suggesting little or no dust reddening in this object.

The balnicity index ${ }^{1}$ is $1950 \pm 250 \mathrm{~km} \mathrm{~s}^{-1}$.

This BAL was observed with XMM by Brandt et al. (2001) who derive a very steep optical-to-X-ray spectral index $\alpha_{\mathrm{OX}}$, which is rather typical of BAL quasars as a consequence of strong gas absorption which suppresses the X-ray emission.

\section{4. $\operatorname{SDSS0756+41~}(z=5.09)$}

The Amici+NICS spectrum of this quasar is shown in Fig. 4, which is significantly noisier with respect to the previous spectra. Our spectrum suggests a slightly lower redshift of 5.08 than previously reported and both CIV and CIII] appear blueshifted with respect to the template. There is a blueshifted absorption $\left(9000 \mathrm{~km} \mathrm{~s}^{-1}\right)$ of CIV with balnicity index of $270 \pm 60 \mathrm{~km} \mathrm{~s}^{-1}$. This absorption is much weaker than in the previous cases, but still quite significant $(5 \sigma)$, also given that the absorption is

\footnotetext{
${ }^{1}$ Goodrich et al. (2001) obtain a much lower balnicity index for this quasar because they integrated starting from $5000 \mathrm{~km} \mathrm{~s}^{-1}$ blueward of the peak, while the formal balnicity index has to be calculated starting $3000 \mathrm{~km} \mathrm{~s}^{-1}$ blueward of the peak (Weymann et al. 1991).
} 

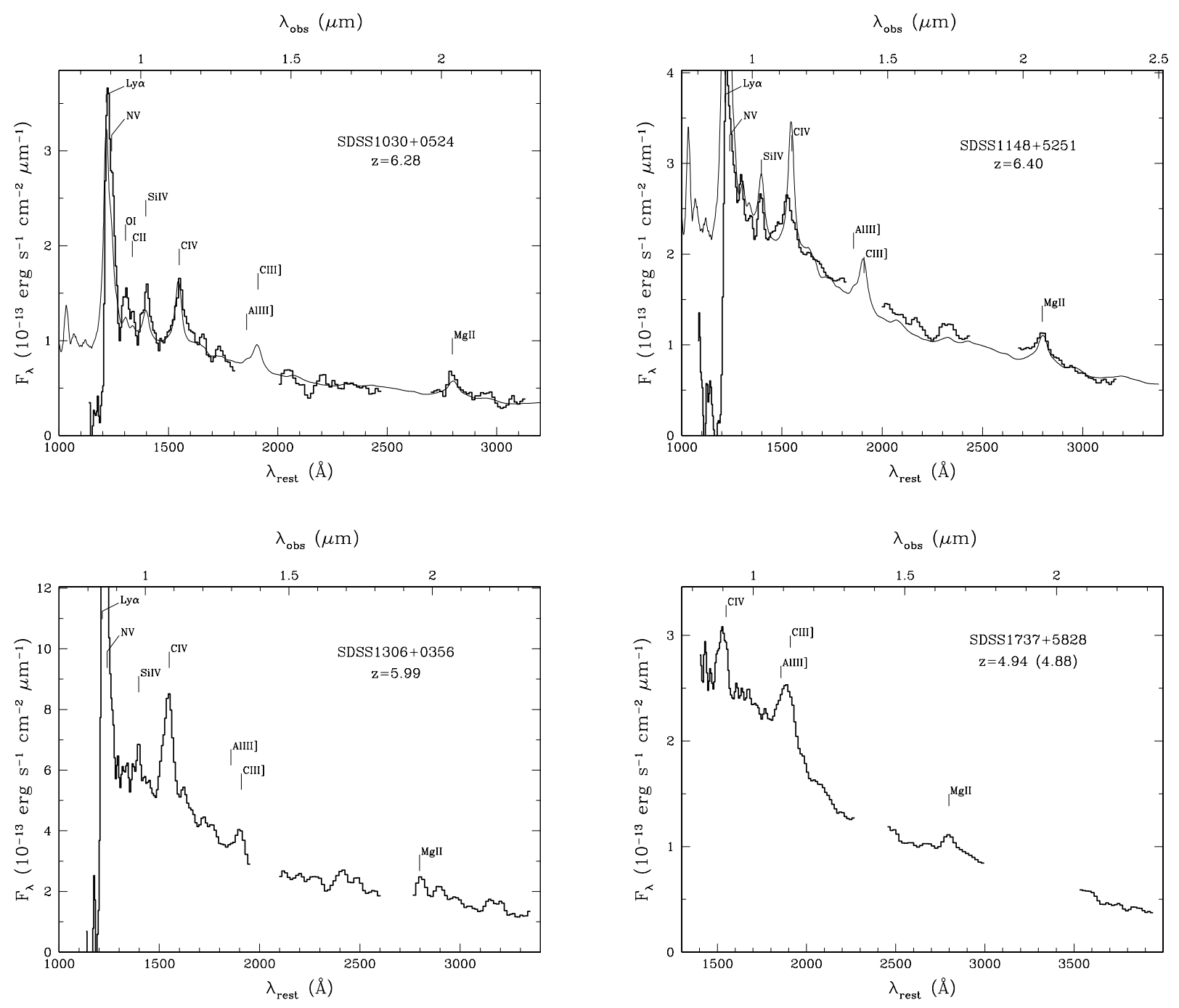

Fig. 5. Amici+Nics spectra of non-BAL quasars at high redshift.

consistently observed in three different spectra (taken on different nights), which were coadded to produce the final spectrum shown in Fig. 4. Some absorption may be observed blueward of MgII, but with a slightly different velocity $\left(6000 \mathrm{~km} \mathrm{~s}^{-1}\right)$. We also find a dip in the blue region of the FeII bump, which might trace Fe absorption and would identify this as a FeLoBAL. However, higher $\mathrm{S} / \mathrm{N}$ spectra are required to confirm this finding. FeLoBAL with weak or no CIV absorption have been found in the SDSS (Reichard et al. 2003a).

The continuum slope is slightly bluer than the non-BAL template $(\alpha=-1.67)$, again indicating no dust reddening.

There is an emission feature at $1800 \AA$, but we note that it may be attributed to imperfect subtraction of a bright sky emission feature in this spectral region.

\section{Non-BAL quasars at $4.9<z<6.4$}

Four out of the eight quasar at $z>4.9$ do not show indication of BAL-like absorption features. Their Amici+NICS spectra are shown in Fig. 5. However the limited spectral resolution (which might cause a blending with the emission lines) and the

limited signal-to-noise do not allow us to exclude the presence of absorption features with a balnicity index below $\sim 200 \mathrm{~km} \mathrm{~s}^{-1}$. As listed in Table 1 the continuum of these nonBAL spectra does not show indication of significant reddening and, actually, they are generally bluer than the average of nonBAL quasars found at lower redshift. This finding suggests that, at least in this small sample, there are no representatives of the reddened quasars found at lower redshift (Richards et al. 2003).

\section{The fraction of BAL quasars at $4.9<z<6.4$}

As mentioned in the Introduction, the fraction of BAL quasars at low redshift is about $15 \%$. Only $2 \%$ of the quasar population are LoBAL, while FeLoBAL quasars are even more rare. Reichard et al. (2003b) obtained that the fraction of BAL quasars is constant up to $z<4$.

The very limited statistics of our sample does not allow us to draw statistically firm conclusions on the fraction of BAL quasars at high redshift. However, it is impressive that out of 8 quasars 4 are BAL. The probability that the fraction of BAL quasars in this small sample is consistent with the fraction of 0.15 found at lower redshift is only $2 \%$. Even 
more astonishing is that two (may be three) of these objects are LoBAL, which is an extremely rare class at low redshift. Note that there are no obvious effects, in terms of spectral shape, that would favor the selection of BAL quasars at $z>5$ in the the SDSS survey (actually any dust reddening would bias against BAL quasars). These findings suggest that at $z \sim 5-6$ the fraction of BAL, and specifically the fraction of LoBAL, increases dramatically. This might be associated with larger amount of (accreting) gas surrounding the circumnuclear region of these high redshift quasars, as discussed in Sect. 8.

Another possibility is that the larger fraction of BALs among high redshift quasars is a consequence of a selection effect. More specifically, high redshift QSOs are more luminous and, therefore, probably accreting at higher rate. If BAL QSOs are associated with high accretion rates, as suggested by various studies (Sects. 1 and 8), an implication would be that they are more likely to be selected at higher redshift. Actually, at $z<4$ where larger samples of quasars and BALs are available, there is no evidence that the BAL fraction changes with luminosity, although dust absorption and variations of the spectral slope (Reichard et al. 2003b) may prevent the detection of such a dependence. However, we have investigated this possibility by comparing the luminosity of our BAL quasars at $z \sim 5-6$ with the luminosity of the BALs at lower redshift in Reichard et al. (2003b). We derived the absolute magnitude $M_{i^{*}}$ in the rest frame $i^{*}$ band (which is tabulated also for the SDSS BAL QSOs at $z<4$ ), by using the extrapolation of the slope measured in our spectra to derive the K-correction. The resulting absolute magnitudes ${ }^{2}$ are reported in Table 1 . If in Reichard et al. (2003b) we select those BALs at $2.5<z<4$, a range where the statistics is still very high and where the fraction of BAL is still estimated to be $\sim 15 \%$, we obtain absolute magnitudes ranging from -26 to -28.8 with an average of $\left\langle M_{i^{*}}\right\rangle_{2.5<z<4}=-27.4$, and a standard deviation $\sigma_{M_{i^{*}}}=0.66$. The comparison with the absolute magnitudes in Table 1 indicates no obvious differences in the luminosity distribution between the sample of BALs at $4.9<z<6.4$ and those at $2.5<z<4$. Table 1 also shows that the non-BAL quasars in our sample are even more luminous than BALs. If one takes into account that BALs at lower redshift tend to be absorbed by dust (which seems not to be the case in our redshift sample, as discussed in Sect. 7) a correction for dust absorption would probably make the low redshift sample even more luminous. Summarizing, a contribution from a luminosity effect on the larger fraction of BALs at high redshift cannot be excluded, but probably it does not play a dominant role.

\section{The balnicity index at $4.9<z<6.4$}

With four BAL quasars only it is not possible to derive a distribution for the CIV balnicity index among quasars at $z>4.9$. Nonetheless, it is most interesting to note that three of these BALs have very high balnicity indices, which are in the high tail of the distribution found for quasars at $z<4$ (Fig. 7 of Reichard et al. 2003a). In particular two of the BAL in our

\footnotetext{
${ }^{2}$ For consistency with Reichard et al. (2003b) we adopt $H_{0}=$ $50 \mathrm{~km} \mathrm{~s}^{-1} \mathrm{Mpc}^{-1}, \Omega_{\mathrm{m}}=1$ and $\Omega_{\Lambda}=0$.
}

sample have a balnicity index higher than $6000 \mathrm{~km} \mathrm{~s}^{-1}$, while at lower redshift $(z<4)$ only $2 \%$ of BAL have balnicity index higher than this value. This result is also indicative of larger amount of circumnuclear gas in these high redshift quasars.

\section{Dust at $4.9<z<6.4$}

One of the most interesting peculiarities of the BAL quasars found at $z>4.9$ is that most of them appear not to be reddened by dust. In particular most of them are bluer than the average slope of the corresponding sub-class at $z<4$ (we recall that at low redshift $\langle\alpha[$ NonBAL $]\rangle=-1.62,\langle\alpha[H i B A L]\rangle=-1.39$, $\langle\alpha[L o B A L]\rangle=-0.93$, Reichard et al. 2003b). There are some deviations from this trend, in particular Stern et al. (2003) have found evidence for significant reddening in one quasar at $z=$ 5.8 and also, within our sample, one of the two LoBAL quasars appears to be significantly reddened (although still bluer than the average of low redshift LoBAL). However, the general trend is to have bluer continuum slopes. The most intriguing case is the most distant LoBAL (SDSS1048+46 at $z=6.22$, Fig. 1) which is much bluer than any LoBAL found at $z<4$.

One naive interpretation would be that at these high redshift we are approaching the epoch when dust had no time to form in large quantities, both because of the lower metallicities and because AGB stars did not have enough time to inject dust into the ISM. Therefore, these high redshift quasars could be relatively poor in dust. On the contrary, powerful dust emission in the $\mathrm{mm} / \mathrm{submm}$ was detected in several of these quasars, and in particular in three of the BAL quasars, indicating that these systems are characterized by very large dust masses (Priddey et al. 2003; Bertoldi et al. 2003).

A possible explanation of this puzzling result may be found in the origin and evolution of dust in these very high redshift quasars. Possibly, the gas density in the circumnuclear region of these primordial quasars is much higher than at lower redshift. Higher gas density may favor the growth of grains and yielding to a flatter extinction curve, which would significantly reduce the reddening by dust. An additional or alternative possibility is that at these extreme redshifts the main dust production mechanism is different. While at low redshift dust is predominantly produced in AGB stars, at very high redshift dust may be predominantly produced by SNe (Schneider et al. 2003; Nozawa et al. 2003; Todini \& Ferrara 2001) or even directly produced in the the BLR of quasars (Elvis et al. 2002): the different dust production mechanism may yield an extinction curve quite different than observed locally. This issue will be discussed more in detail in a forthcoming paper (Maiolino et al. in preparation).

\section{Discussion}

Although the small number of objects does not allow us to draw strong conclusions, our results suggest that the physics of the circumnuclear medium of the most distant quasars currently known, at $4.9<z<6.4$, is probably different than in lower redshift quasars. The large fraction of BAL quasars suggests that either the outflowing wind occurs over a much wider solid 
angle (in a scenario where BAL and nonBAL quasars are unified through the viewing angle) or that the fraction of quasars experiencing strong outflowing winds is much larger at high redshift (in a scenario where the wind of BAL quasars covers isotropically the continuum source). Both scenarios would imply an evolution of quasars' circumnuclear medium at high redshift. Both the extreme depth of the absorption features and the identification of low ionization species (MgII, AlIII]) suggest that the column density of the outflowing gas is higher in these high redshift quasars. Summarizing, quasars at $4.9<z<6.4$ are probably expelling larger amount of gas, through winds, than quasars at $z<4$.

As suggested by some authors (e.g., King 2003; King \& Pounds 2003; Mathur 2001) the strong winds observed in some AGN are probably associated with large accretion rates. In particular, quasars characterized by the strongest outflows (in terms of mass outflow rate) may be accreting at or above the Eddington rate. Within this context, the differences found by us between low and high redshift quasars may reflect different evolutionary stages, where the most distant quasars are accreting at a significantly higher rate. Such an evolutionary scenario for these highest redshift BAL quasars would also be consistent with the study of Becker et al. (2000) on radio loud quasar, who find that the BAL phenomenon is preferentially associated with younger radio sources and, therefore, tracing the early stages of quasar activity. Recently Willott et al. (2003) and Lewis et al. (2003) suggested that the evolutionary scenario for BALs might be wrong based on the lack of difference in terms of sub-mm emission between non-BALs and BALs. However, they only probed the redshift range $1.0<z<2.6$. As mentioned by Willott et al. (2003), their finding may still fit within the evolutionary scenario if the sub-mm emission by the host galaxy is not related to the BAL activity of the nucleus, at least in the $1.0<z<2.6$ redshift range.

At $z \sim 6$ we are probably probing the epoch of formation for the first galaxies, and approaching the re-ionization epoch, as inferred by the evolved metallicity of these systems (e.g., Maiolino et al. 2003; Dietrich et al. 2003; Pentericci et al. 2002). Our results would support the scenarios of a coevolution between QSOs and host galaxies, which predict that the rapidly forming spheroids at high redshift would be associated with a large accretion rate onto the central Black Hole as a consequence of the enhanced viscous drag (e.g., Granato et al. 2004; Umemura et al. 1997). The same models expect a strong feedback by the quasars themselves in the form of strong outflows, as actually observed by us. Although the qualitative agreement between observations and models is remarkable, much effort is still required for an accurate quantitative comparison.

\section{Conclusions}

We have presented near-IR spectra covering the whole $0.8-2.4 \mu \mathrm{m}$ range for eight among the most distant quasars known, and specifically in the redshift range $4.9<z<6.4$. At these redshifts our spectra sample the UV rest-frame spectral region and, in particular, include several prominent UV lines such as CIV, AlIII], CIII], MgII and the UV FeII bump. The observational results strongly suggest that the physical properties of the circumnuclear medium of these most distant quasars are different with respect to lower redshift quasars. More specifically, the observational results can be summarized as follows:

1. Half of the quasars show broad and deep (blueshifed) absorption of CIV, i.e. belong to the class Broad Absorption Line (BAL) quasars, which are characterized by large quantities of gas outflowing at high velocities. At lower redshift $(z<4)$ the fraction of BAL quasars is about $15 \%$. Our finding strongly suggests that the fraction of BAL quasars increases at $z>4.9$. We cannot exclude a possible luminosity dependence of the BAL fraction, although probably it does not play a major role.

2. Two (possibly three) of the BAL quasars also show absorption associated with low ionization lines (MgII and AlIII]), i.e. belong to the subclass of "Low Ionization BAL" (LoBAL). This class of quasars is very rare at lower redshifts, accounting only for $2 \%$ of the whole quasar population at $z<4$. Our finding suggests that LoBAL may be much more common at $z>4.9$.

3. The CIV absorption features are on average deeper than found in lower redshift BAL quasars. This finding, along with the presence of low ionization species in absorption, indicates that the column density of the outflowing gas is larger than in lower redshift quasars.

4. The most distant BAL quasar (SDSS1048+46, a LoBAL at $z=6.22$ ) is also characterized by an absorption feature on the red side of CIV. This might be absorption due to redshifted CIV, or blueshifted absorption associated with HeII $\lambda 1640$ (observed only in one case at lower redshift).

5. The continuum of the quasars in our sample is on average bluer than the quasars at lower redshift. The most interesting case is the most distant LoBAL quasar which is bluer than any quasar of the same class found at lower redshift (although an intriguing bending of the spectrum at $\lambda<1500$ is observed). These findings suggest little, or very low, dust reddening with respect to what observed in lower redshift quasars. Yet, dust is present in large quantities, as inferred by the $\mathrm{mm} / \mathrm{submm}$ detections.

These observational results suggest the following conclusions about the physics of the most distant quasars:

i) The large fraction of BAL quasars, the depth and ionization state of the absorption systems suggest that, at $z \sim 5-6$, quasars are surrounded by larger amount of denser gas with respect to lower redshift quasars.

ii) The presence of strong outflows is likely associated with high accretion rates in these primordial quasars. Strong quasars winds and high accretion rates are expected by the models of co-evolution of quasars and host galaxies at high redshifts.

iii) The dust extinction curve in these distant quasars is probably different than lower redshift quasars. This may reflect different evolution and formation mechanisms of dust grains at $z>5$. This issue will be discussed more in detail in a forthcoming paper (Maiolino et al., in preparation). 
Acknowledgements. We thank G. Richards and P. Hall for very useful comments. This work was partially supported by the Italian Ministry of Research (MIUR) and by the National Institute for Astrophysics (INAF).

\section{References}

Becker, R. H., White, R. L., Gregg, M. D., et al. 2000, ApJ, 538, 72 Baffa, C., Comoretto, G., Gennari, S., et al. 2001, A\&A, 378, 722 Bertoldi, F., Carilli, C. L., Cox, P., et al. 2003, A\&A, 406, L55 Boroson, T. A., \& Meyers, K. A. 1992, ApJ, 397, 442

Brandt, W. N., Guainazzi, M., Kaspi, S., et al. 2001, AJ, 121, 591

Brotherton, M. S., Tran, H. D., Becker, R. H., et al. 2001, ApJ, 546, 775

Dietrich, M., Hamann, F., Appenzeller, I., \& Vestergaard, M. 2003, ApJ, 596, 817

Elvis, M. 2000, ApJ, 545, 63

Elvis, M., Marengo, M., \& Karovska, M. 2002, ApJ, 567, L107

Fan, X., Strauss, M. A., Schneider, D. P., et al. 2003, AJ, 125, 1649

Francis, P. J., Hewett, P. C., Foltz, C. B., et al. 1991, ApJ, 373, 465

Gaskell, C. M., Goosmann, R. W., Antonucci, R. R. J., et al. 2003, ApJ, submitted [arXiv: astro-ph/0309595]

Goodrich, R. W., Campbell, R., Chaffee, F. H., et al. 2001, ApJ, 561, L23

Granato, G. L., Silva, L., De Zotti, G., Bressan, A., \& Danese, L. 2003, ApJ, 600, 58

Hall, P. B., Anderson, S. F., Strauss, M. A., et al. 2002, ApJS, 141, 267

Hall, P. B., Knapp, G. R., Richards, G. T., et al. 2004, in Multiwavelength AGN Surveys, ed. R. Mujica, \& R. Maiolino (World Scient. Publ.), in press [arXiv: astro-ph/0403347]

Hamann, F., \& Ferland, G. 1999, ARA\&A, 37, 487

Hewett, P. C., \& Foltz, C. B. 2003, AJ, 125, 1784

King, A. 2003, ApJ, 596, L27

King, A. R., \& Pounds, K. A. 2003, MNRAS, 345, 657
Lewis, G. F., Chapman, S. C., \& Kuncic, Z. 2003, ApJ, 596, L35

Maiolino, R., Marconi, A., Salvati, M., et al. 2001a, A\&A, 365, 28

Maiolino, R., Marconi, A., \& Oliva, E. 2001b, A\&A, 365, 37

Maiolino, R., Mannucci, F., Baffa, C., Gennari, S., \& Oliva, E. 2001c, A\&A, 372, L5

Maiolino, R., Juarez, Y., Mujica, R., Nagar, N. M., \& Oliva, E. 2003, ApJ, 596, L155

Mathur, S. 2001, AJ, 122, 1688

Nozawa, T., Kozasa, T., Umeda, H., et al. 2003, ApJ, in press [arXiv: astro-ph/0307108]

Ogle, P. M., Cohen, M. H., Miller, J. S., et al. 1999, ApJS, 125, 1

Oliva, E. 2003, Mem. Soc. Astron. It., 74, 118

Pentericci, L., Fan, X., Rix, H.-W., et al. 2002, AJ, 123, 2151

Priddey, R. S., Isaak, K. G., McMahon, R. G., Robson, E. I., \& Pearson, C. P. 2003, MNRAS, 344, L74

Pickles, A. J. 1998, PASP, 110, 863

Reichard, T. A., Richards, G. T., Schneider, D. P., et al. 2003a, AJ, 125,1711

Reichard, T. A., Richards, G. T., Hall, P. B., et al. 2003b, AJ, 126, 2594

Richards, G. T., Hall, P. B., Vander Berk, D. E., et al. 2003, AJ, 126, 1131

Schmidt, G. D., \& Hines, D. C. 1999, ApJ, 512, 125

Schneider, R., Ferrara, A., \& Salvaterra, R. 2003, MNRAS [arXiv: astro-ph/0307087]

Stern, D., Hall, P. B., Barrientos, L. F., et al. 2003, ApJ, 596, L39

Surdej, J., \& Hutsemekers, D. 1987, A\&A, 177, 42

Todini, P., \& Ferrara, A. 2001, MNRAS, 325, 726

Umemura, M., Fukue, J., \& Mineshige, S. 1997, ApJ, 479, L97

Verner, E., Bruhweiler, F., Verner, D., Johansson, S., \& Gull, T. 2003, ApJ, 592, L59

Weymann, R. J., Morris, S. L., Foltz, C. B., \& Hewett, P. C. 1991, ApJ, 373,23

Willott, C. J., Rawlings, S., \& Grimes, J. A. 2003, ApJ, 598, 909 